\title{
Carcinoma of Littre Glands
}

National Cancer Institute

\section{Source}

National Cancer Institute. Carcinoma of Littre Glands. NCI Thesaurus. Code C39865.

An adenocarcinoma that arises from the Littre glands. 